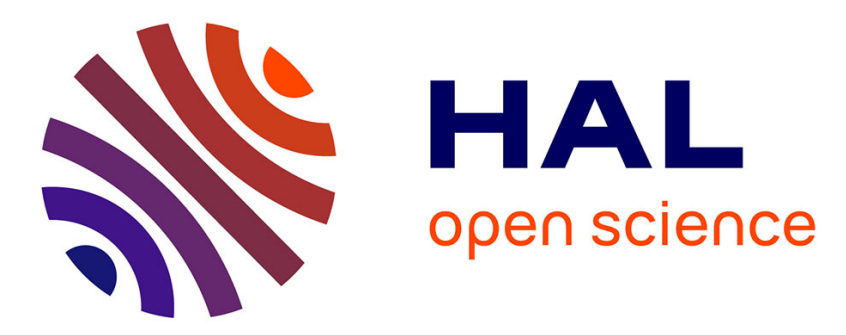

\title{
Rheological characterization of mechanical properties of chemically crosslinked microspheres
}

\author{
van Nga Nguyen, Nicolas Huang, Jean-Louis Grossiord, Laurence Moine, \\ Florence Agnely, Christine Vauthier
}

\section{- To cite this version:}

van Nga Nguyen, Nicolas Huang, Jean-Louis Grossiord, Laurence Moine, Florence Agnely, et al.. Rheological characterization of mechanical properties of chemically crosslinked microspheres. Journal of Applied Polymer Science, 2012, 128 (5), pp.3113 - 3121. 10.1002/app.38510 . hal-03199935

\section{HAL Id: hal-03199935 \\ https://hal.science/hal-03199935}

Submitted on 16 Apr 2021

HAL is a multi-disciplinary open access archive for the deposit and dissemination of scientific research documents, whether they are published or not. The documents may come from teaching and research institutions in France or abroad, or from public or private research centers.
L'archive ouverte pluridisciplinaire HAL, est destinée au dépôt et à la diffusion de documents scientifiques de niveau recherche, publiés ou non, émanant des établissements d'enseignement et de recherche français ou étrangers, des laboratoires publics ou privés. 


\title{
Rheological characterisation of mechanical properties of chemically crosslinked microspheres
}

\author{
Van Nga Nguyen ${ }^{1,2}$, Nicolas Huang ${ }^{1,3}$, Jean-Louis Grossiord ${ }^{1,3}$, Laurence \\ Moine $^{1,3}$, Florence Agnely ${ }^{1,3}$, Christine Vauthier ${ }^{1,3 *}$.
}

${ }^{1}$ Univ Paris-Sud, Faculté de Pharmacie, 5, rue J.B. Clément, 92296 Châtenay-Malabry, France ; ${ }^{2}$ Occlugel SARL, Paris BioTech, 24 Rue du Faubourg Saint-Jacques, Paris, F-75014 ; ${ }^{3}$ CNRS UMR 8612, Institut Galien Paris-Sud, 5, rue J.B. Clément, 92296 Châtenay-Malabry, France

Published in J App/ Polym Sci 2013; 128(5):3113-3121. https://doi.org/10.1002/app.38510

Received 11 May 201; Accepted 19 August 2012; Published online 17 September 2012.

Corresponding author: Christine Vauthier, Institut Galien Paris-Sud, UMR CNRS 8612, Université Paris-Sud, Faculté de Pharmacie, 5 rue J.B. Clément, 92296 CHATENAY-MALABRY Cedex France. christine.vauthier@u-psud.fr
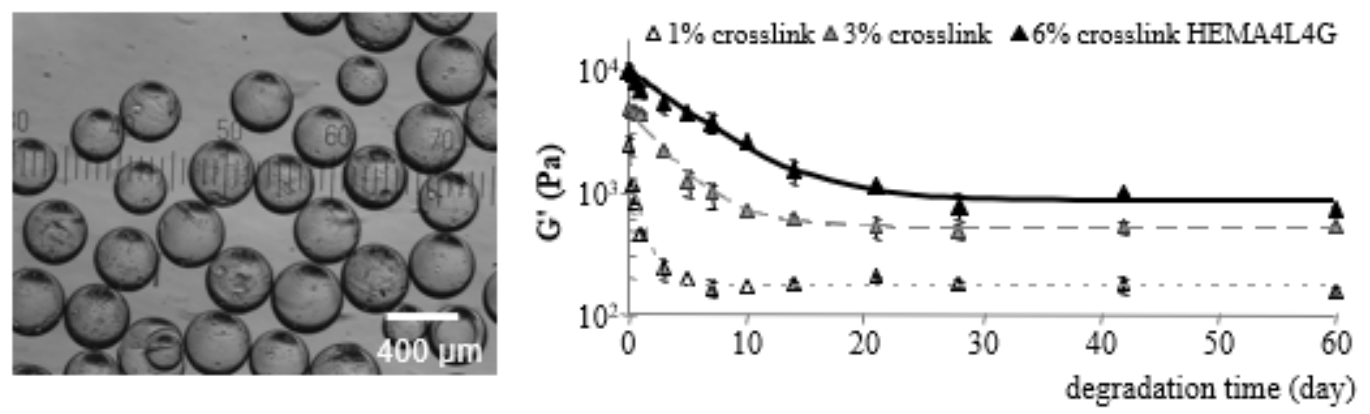

\begin{abstract}
This work presents a rheological method to characterize degradation of microspheres made of chemically crosslinked hydrogels. Conditions to measure rheological properties of microspheres remaining as individual microspheres during measurement were established. Relevant and reproducible measurements could be obtained with a rheometer equipped with a parallel plate geometry in which a homogenous and continuous monolayer of microspheres was inserted in the gap between the plates. The storage modulus of microspheres was determined under an imposed strain of $0.04 \%$ with an oscillatory measurement mode at various frequencies. The microspheres showed almost purely elastic behaviour while their storage modulus was affected by the degree of crosslinking. In the second part of the work, the rheological method was applied to investigate the degradation of microspheres made of a hydrolysable crosslinked hydrogel. The method was found suitable by measuring the storage modulus over time. A good correlation was identified between acidification of the incubation medium due to the release of degradation products and the decrease of the storage modulus of the microspheres indicating a reduction of the crosslinking of the hydrogel resulting form the degradation process.
\end{abstract}

Keywords: rheology, crosslink, hydrogels, microsphere, degradation 


\section{INTRODUCTION}

Hydrogels are the synthetic biomaterial class the most similar to natural living material $[1,2]$. They consist of three-dimensional crosslinked macromolecular networks with a capacity of water absorbing up to thousand times their dry weight without dissolving [3,4]. This unique property contributes to their soft consistency and biocompatibility. Therefore, they are receiving significant attention in developing biomaterials for medical applications including tissue engineering, cell encapsulation and drug delivery [5-9]. For many of the applications in the biomedical field, the mechanical properties of hydrogels should be appropriate.

Rheology is one of the most employed methods used to characterise gels from their formation to their intimate properties. Indeed, rheological properties are sensitive to the degree of crosslinking of the material and were widely used to follow the kinetic of crosslinking reaction used during formation of chemically crosslinked hydrogels [10-13]. Various experimental conditions are suitable to study rheological properties of hydrogel. The most used include time sweep at constant frequency and strain [10-12] or frequency sweep at constant shear stress amplitude [13]. Forming hydrogels can be also characterized by different rheological methods i.e., frequency sweep, strain sweep or stress sweep mode $[14,15]$. It is noteworthy that these methods were mainly applied to characterize bulk gels while their application to monitor degradation of hydrogels including chemical crosslinks remains marginal. This is contradictory to the fact that rheological properties of hydrogels are sensitive to the degree of crosslinking and are suitable to follow gel formation during crosslinking reaction. Thus, it can be assumed that it could also be used more systematically to monitor a decrease of the crosslinking ratio occurring during degradation of a hydrolysable crosslinked hydrogel. Only a few studies have applied rheological methods to follow degradation of hydrogels caused either from a biodegradable polymer network $[16,17]$ or by the association of opposite charged microspheres [18]. In all cases, it was demonstrated that the rheological properties of the gels were modified during degradation and that the methods were suitable to monitor the degradation of such materials. This is an advantage over all the other methods of characterisation of polymers such as GPC, DSC, ${ }^{1} \mathrm{H}$ NMR which can not be applied to characterize materials made of three-dimensional polymer networks since they are not soluble in any solvent.

Rheology is a powerful tool for the characterization of bulk hydrogels. However, it is noteworthy that its application to the characterization of hydrogels under the form of nano- and microparticles has led to a very limited number of works so far [19]. The aim of our work was to develop a method based on rheological measurements that would be suitable to characterize hydrogels occurring as individual microspheres and to follow their degradation by hydrolysis of their crosslinking bonds. The first part of the work describes the set up and experimental conditions resulting into reproducible measurements of the rheological characteristics of hydrogel microspheres with different degrees of reticulation. In the second part of the work, the rheological method was applied to monitor the degradation of hydrogel microspheres containing hydrolysable crosslinking bonds.

\section{EXPERIMENTAL}

\subsection{Materials}

Poly(ethylene glycol methyl ether methacrylate) (PEGMMA) of number-average molecular weight $300 \mathrm{~g} / \mathrm{mol}$, poly(ethylene glycol dimethacrylate) (PEGDMA) of number-average molecular weight 575 $\mathrm{g} / \mathrm{mol}$, poly(vinyl alcohol) (PVA) $\left(\mathrm{MW}_{\mathrm{n}} 89,000-98,000 \mathrm{~g} / \mathrm{mol} 99+\%\right.$ hydrolyzed) were purchased from Sigma-Aldrich (St. Louis, USA). Molar mass values were provided by supplier. Azobisisobutyronitrile 
(AIBN) used as polymerization initiator was obtained from Acros Organic (Geel, Belgium). Analytical grade solvents were supplied by Carlo Erba (Val de Rueil, France). All chemicals were used as purchased without further purification. Phosphate buffer saline (PBS) $(58 \mathrm{mM}, 150 \mathrm{mM} \mathrm{NaCl})$ at pH 7.4 was used as incubation medium for the microsphere degradation study. ${ }^{1} \mathrm{H}$ NMR analyses were performed with a $300 \mathrm{MHz}$ Bruker apparatus. Deuterium solvents are purchased from Carlo Erba.

\subsection{Methods}

\subsubsection{Synthesis of ethylene glycol-co-tetralactic-co-tetraglycolic dimethacrylate (HEMA4L4G)}

The chemical structure of ethylene glycol-co-tetralactic-co-tetraglycolic dimethacrylate (HEMA4L4G) is given in Figure 1.

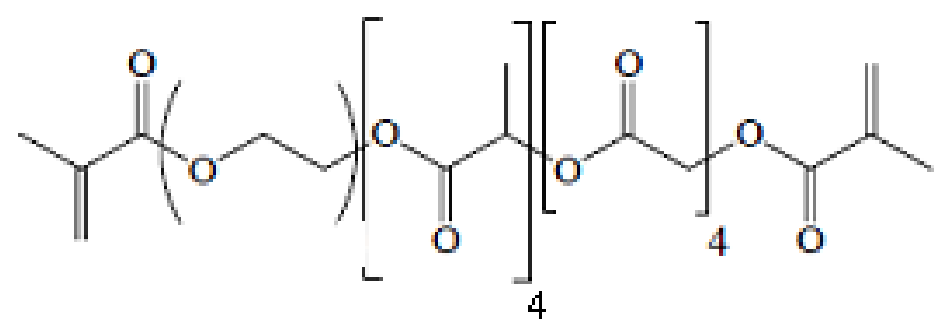

Figure 1: Chemical structure of the hydrolysable crosslinker Ethylene glycol-co-tetralactic-cotetraglycolic dimethacrylate (HEMA4L4G) used in the synthesis of degradable microspheres

In a dry round flask containing a magnetic stir bar, lactide $(8 \mathrm{mmol}, 1.152 \mathrm{~g})$, glycolide $(8 \mathrm{mmol}, 0.929$ g), HEMA ( $4 \mathrm{mmol}, 0.52 \mathrm{~g}$ ) and $\mathrm{Sn}(\mathrm{Oct})_{2}(0.025 \mathrm{mmol}, 10 \mathrm{mg})$ were dissolved in toluene $(5 \mathrm{~mL})$ under an argon atmosphere. After $20 \mathrm{~h}$ at $90^{\circ} \mathrm{C}$, chloroform $(5 \mathrm{~mL})$ was added to dilute the reaction mixture and the resulting polymer was purified by precipitating in a large volume of petroleum ether. The precipitate was then dried under vacuum.

${ }^{1} \mathrm{H}$ NMR $\left(\mathrm{CDCl}_{3}\right) \mathrm{d}(\mathrm{ppm}): 6.13(\mathrm{~s}, 1 \mathrm{H}, \mathrm{CHH}=), 5.58(\mathrm{~s}, 1 \mathrm{H}, \mathrm{CHH}=), 5.27\left(\mathrm{~m}, 1 \mathrm{H} \times \mathrm{PD}_{\mathrm{L}}, \mathrm{PLA}\right), 4.73(\mathrm{~m}, 2 \mathrm{H} \times$ $\left.\mathrm{PD}_{\mathrm{G}}, \mathrm{PGA}\right), 4.44$ ( $\left.\mathrm{m}, 2 \times \mathrm{CH}_{2}, \mathrm{HEMA}\right), 1.95$ (s, 3H, methacrylate), 1.59 ( $\left.\mathrm{m}, 3 \mathrm{H} \times \mathrm{PD}_{\mathrm{L}}, \mathrm{PLA}\right)$. According to the NMR result, the polymerization degree of lactic $P D_{L}$ was around 4 . The same value was found for the polymerization degree of glycolic $\mathrm{PD}_{\mathrm{G}}$.

The dried polymer obtained from the first step was then subjected to an esterification on the hydroxyl group at the end of the PLGA chain by reacting with methacryloyl chloride. The total amount of the forming macromer was dissolved in $\mathrm{CH}_{2} \mathrm{Cl}_{2}(30 \mathrm{ml})$ under argon in a dry flask equipped with magnetic stir bar. The content of the flask was cooled to $0^{\circ} \mathrm{C}$ and TEA $(12 \mathrm{mmol}, 1.6 \mathrm{~mL})$ was added in order to trap $\mathrm{HCl}$ formed during the esterification reaction. The solution was stirred and then methacryloyl chloride ( $12 \mathrm{mmol}, 1.2 \mathrm{~mL}$ ) was added dropwise to the solution during $30 \mathrm{~min}$. The stirring was continued $1 \mathrm{~h}$ at $0^{\circ} \mathrm{C}$ and then $24 \mathrm{~h}$ at room temperature. The triethylamine chloridrate salt resulting from TEA and $\mathrm{HCl}$ was removed by filtration and the polymer was precipitated in a large volume of petroleum ether. The crude product was then separated, dried, and kept in an argon atmosphere for further reactions.

The methacrylate functionality $f$ of the macromonomer, the polymerization degree of lactic acid and of glycolic acid (respectively $\mathrm{PD}_{\mathrm{L}}$ and $\mathrm{PD}_{\mathrm{G}}$ ) were determined from ${ }^{1} \mathrm{H}$ NMR spectra of the obtained products. ${ }^{1} \mathrm{H} \mathrm{NMR}\left(\mathrm{CDCl}_{3}\right) \mathrm{d}(\mathrm{ppm}): 6.22(\mathrm{~m}, 2 \mathrm{H} \times f, \mathrm{CHH}=), 5.64(\mathrm{~m}, 2 \mathrm{H} \times f, \mathrm{CHH}=), 5.18\left(\mathrm{~m}, 1 \mathrm{H} \times \mathrm{PD}_{\mathrm{L}}\right.$, 
PLA), $4.82\left(\mathrm{~m}, 2 \mathrm{H} \times \mathrm{PD}_{\mathrm{G}}, \mathrm{PGA}\right), 4.31(\mathrm{~m}, 4 \mathrm{H}, \mathrm{TEG}), 3.64-3.70(\mathrm{~m}, 12 \mathrm{H}, \mathrm{TEG}), 1.97(\mathrm{~m}, 6 \mathrm{H} \times f$, methacrylate), $1.53\left(\mathrm{~m}, 3 \mathrm{H} \times \mathrm{PD}_{\mathrm{L}}, \mathrm{PLA}\right) . \mathrm{PD}_{\mathrm{L}}$ and $\mathrm{PD}_{\mathrm{G}}$ were around 4 . The $f$ value was $97 \%$.

\subsubsection{Synthesis of crosslinked hydrogel microspheres}

A $0.75 \%$ solution of PVA $(300 \mathrm{~mL})$ was introduced into a $500 \mathrm{~mL}$ reactor and was purged with nitrogen atmosphere for $15 \mathrm{~min}$. The dispersed phase containing the crosslinking agent and a comonomer, PEGMMA, at various molar ratios were solubilized in $12 \mathrm{~mL}$ of toluene and degassed by bubbling nitrogen through the solution for $15 \mathrm{~min}$. Non degradable microspheres were synthesised with PEGDMA as crosslinking agent at different molar ratios (expressed as percentage of the total monomers) ranging from 1 to $11 \%$ (Table I). Degradable microspheres were synthesized using $3 \%$ (in molar ratio regarding total amount of monomers) of HEMA4L4G as the crosslinking agent. Preparation of the microspheres was continued as followed in all cases. The dispersed phase containing the co-monomers dissolved in toluene was introduced into the aqueous phase at $30^{\circ} \mathrm{C}$ and agitated by means of a homemade glass 3-wing-propeller type stirrer at $250 \mathrm{rpm}$. AIBN $(0.3 \mathrm{~g})$ was solubilized in $2 \mathrm{~mL}$ of toluene and was then added into the reactor. The temperature was increased to $70^{\circ} \mathrm{C}$ and the reaction was allowed to proceed under stirring for $15 \mathrm{~h}$ at $70^{\circ} \mathrm{C}$. The microspheres were washed with acetone and water before they were sieved over the following series of sieves (Inox sieve with mesh size 630, 500, 315, 100, $40 \mu \mathrm{m}$ ) (Fisher scientific, Illkirch, France). Particles retained by the sieve with a mesh size of $315 \mu \mathrm{m}$ corresponding to microspheres with diameter ranging from 315 to $500 \mu \mathrm{m}$ were kept for the present study. They were freeze dried immediately after preparation and purification and stored at $-20^{\circ} \mathrm{C}$ until use.

Table I Characteristics of microspheres obtained after sieving between 315 and $500 \mu \mathrm{m}$.

\begin{tabular}{|c|c|c|c|c|c|c|}
\hline Sample name & $\begin{array}{l}\text { crosslinking } \\
\text { molar ratio } \\
(\%)\end{array}$ & $\begin{array}{l}\text { nature of } \\
\text { crosslink }\end{array}$ & $\begin{array}{l}D[4,3] \\
(\mu \mathrm{m})\end{array}$ & $\begin{array}{l}\text { Span } \\
10^{-1}\end{array}$ & $\begin{array}{l}\mathrm{G}^{\prime} \text { at } 10 \mathrm{~Hz} \\
10^{3}(\mathrm{~Pa})\end{array}$ & $\begin{array}{l}\delta \text { at } 10 \mathrm{~Hz} \\
\left({ }^{\circ}\right)\end{array}$ \\
\hline PEGDMA-1\% & 1 & \multirow{10}{*}{$\begin{array}{l}\text { PEGDMA } \\
\text {-(non } \\
\text { degradable) }\end{array}$} & $462 \pm 2$ & $3.5 \pm 0.1$ & $1.1 \pm 0.1$ & $7.9 \pm 1.8$ \\
\hline PEGDMA-2\% & 2 & & $439 \pm 2$ & $4.0 \pm 0.1$ & $2.8 \pm 0.1$ & $5.6 \pm 0.4$ \\
\hline PEGDMA-2.5\% & 2.5 & & $418 \pm 3$ & $5.0 \pm 0.1$ & $3.8 \pm 0.2$ & $4.2 \pm 0.2$ \\
\hline PEGDMA-3\% & 3 & & $419 \pm 1$ & $4.6 \pm 0.1$ & $5.1 \pm 0.7$ & $4.3 \pm 0.2$ \\
\hline PEGDMA-4\% & 4 & & $391 \pm 2$ & $2.1 \pm 0.1$ & $8.0 \pm 0.4$ & $3.6 \pm 0.6$ \\
\hline PEGDMA-5\% & 5 & & $389 \pm 4$ & $2.3 \pm 0.3$ & $8.7 \pm 0.1$ & $4.4 \pm 0.4$ \\
\hline PEGDMA-6\% & 6 & & $412 \pm 2$ & $4.8 \pm 0.1$ & $12 \pm 1$ & $4.1 \pm 0.3$ \\
\hline PEGDMA-7\% & 7 & & $423 \pm 1$ & $5.0 \pm 0.1$ & $15 \pm 1$ & $3.0 \pm 0.3$ \\
\hline PEGDMA-9\% & 9 & & $406 \pm 2$ & $5.8 \pm 0.1$ & $16 \pm 1$ & $3.8 \pm 0.2$ \\
\hline PEGDMA-11\% & 11 & & $376 \pm 1$ & $2.7 \pm 0.2$ & $17 \pm 1$ & $4.7 \pm 0.6$ \\
\hline HEMA4L4G-3\% & 3 & $\begin{array}{l}\text { HEMA4L4G } \\
\text { (hydrolysable) }\end{array}$ & $379 \pm 2$ & $7.3 \pm 0.1$ & $4.8 \pm 0.2$ & $7.7 \pm 0.7$ \\
\hline
\end{tabular}




\subsubsection{Degradation studies}

Samples of dried microspheres $(0.100 \pm 0.002 \mathrm{~g})$ were suspended in $15 \mathrm{~mL}$ of PBS pH 7.4 and incubated at $37^{\circ} \mathrm{C}$ under lateral stirring at $100 \mathrm{rpm}$ (Incubator shaker KS4000i - IKA). Three essays were performed for each time point $(0,1,3,5,10,14$ and 21 days). At the different incubation times, the supernatant and the microspheres were separated for $\mathrm{pH}$ and rheological measurements, respectively.

\subsubsection{Characterizations of microspheres}

\subsubsection{Morphology and size analysis}

Microspheres obtained after synthesis or during the degradation process were observed by optical microscopy. The optical microscope (OLYMPUS BH2 Microscope) was equipped with leitz PL2.5/0.08 and Olympus Dplan 10 objectives and a Mightex camera. At less 25 micrographs were taken for each sample.

Particle size distribution was determined by laser diffraction on Mastersizer $\mathrm{S}$ apparatus (Malvern Instrument Ltd.) at $25^{\circ} \mathrm{C}$. Dry beads were dispersed in water and were allowed to swell for $15 \mathrm{~min}$ before measurement. This time was sufficient to reach the swelling equilibrium with the type of microspheres considered in the present study. They were then introduced in the QSpec small volume sample dispersion unit. Homogenous circulation between the latter and the measurement cell was performed by means of $1000 \mathrm{rpm}$ magnetically stirring. The quantity of microspheres was added in order to obtain an aperture between 5 and $10 \%$. Each injection was analyzed 3 times. Granulometry was analyzed using the Fraunhofer optical model. Results were presented in \% volume distribution using the volume/mass moment mean diameter $\mathrm{D}[4,3]$ (equation 1 ) and the span of their size distribution (equation 2)

$$
\begin{aligned}
& D[4,3]=\frac{\sum n_{i} d_{i}^{4}}{\sum n_{i} d_{i}^{3}} \\
& \text { Span }=\frac{D[v, 90]-D[v, 10]}{D[v, 50]}
\end{aligned}
$$

With " $n_{i}$ » et $" d_{i}$ » represented the number of particles with a define diameter, $D[v, 90]$ is the volume diameter above which it includes $90 \%$ of the distribution, $D[v, 50]$ is the volume diameter above which it includes $50 \%$ of the distribution, $D[v, 10]$ is the volume diameter above which it includes $10 \%$ of the distribution.

\subsubsection{Mass fraction measurements of microspheres}

The mass fraction of the microspheres in concentrated suspensions obtained after sedimentation was determined as followed. A weighting boat was prepared with a pre-weighted piece of filter paper above which was placed a pre-weighted piece of organza. A sample of the concentrated suspension of microspheres obtained by sedimentation was placed over the organza. The mass measured, $W_{\text {sed }}$, corresponded to the weight of the wet microspheres $\left(W_{W M}\right)$ and the weight of the surrounded liquid. As organza showed a mesh size much below the size of the smaller microspheres and it did not absorb water, it retained the microspheres while the surrounded liquid was absorbed 
by the filter paper placed below. By weighting separately, the pre-weighted piece of organza with the microspheres and the pre-weighted piece of filter paper having absorbed the liquid surrounding the microspheres, it was possible to determine the weight of wet microspheres $\left(W_{W M}\right)$ and that of the surrounding liquid. The mass fraction of the microspheres $\left(f_{w m}\right)$ expressed as a percentage can then be calculated from equation 3 .

$$
f_{w m}=\frac{W_{W M} \times 100}{W_{\text {Sed }}}(\% \mathrm{wt})
$$

\subsubsection{Determination of the Swelling ratio of the microspheres}

The same procedure as described above was used to evaluate the weight of the wet microspheres $\left(W_{W M}\right)$. Then the isolated wet microspheres were freeze dried and weighted again after drying to measure their dried weight $\left(W_{D M}\right)$. The mass swelling ratio, $Q_{w}$, was then calculated from equation 4 :

$Q_{w}=\frac{W_{W M}-W_{D M}}{W_{D M}}$

\subsubsection{4 pH measurements}

The $\mathrm{pH}$ of each supernatant obtained during degradation process was measured with a SevenMulti $\mathrm{pH}$ meter (Mettler Toledo) at $25^{\circ} \mathrm{C}$.

\subsubsection{Rheological measurements}

The rheological properties of microspheres were evaluated by a Haake RheoStress 600 rheometer (Thermo Electron) equipped with a $35 \mathrm{~mm}$ plate-plate geometry. Measurements were performed at $25^{\circ} \mathrm{C}( \pm 0.02)$ and regulated with a Peltier plate. A solvent trap placed on the geometry was used to prevent water evaporation during measurements. Microspheres were allowed to sediment by gravity for 30 min prior sampling. The sediment with a mass fraction in microspheres of $65 \pm 3 \%$ was deposited on the inferior plate of the measurement cell.

Rheological experiments were performed using the oscillatory modes with an imposed strain. The characterization of the microspheres included two steps that were carried out successively including a shear sweep and a frequency sweep. Three rheological parameters were monitored during each measurement which included the storage (or elastic) modulus $G^{\prime}$, the loss (or viscous) modulus $G^{\prime \prime}$ and the phase angle $\delta$ (defined as $\tan \delta=G^{\prime \prime} / G^{\prime}$ ).

At first, a shear sweep was performed to define the stable zone (plateau) of $G^{\prime}$ and $G^{\prime \prime}$ moduli as a function of the applied strain (from 0.0001 to 0.1 ) at a constant frequency of the oscillation $(1 \mathrm{~Hz})$. This experiment was performed on non degradable microspheres with a crosslinking ratio ranging between 1 and $11 \%$. It allowed choosing a strain value in the linear viscoelastic regime which was common for all systems.

Then $G^{\prime}, G^{\prime \prime}$ moduli and phase angle $\delta$ were determined at different frequencies of the oscillation $(0.01-100 \mathrm{~Hz})$ for the different types of non degradable microspheres. In the case of the degradable microspheres, the three rheological parameters were recorded on different aliquots of the same batch of microspheres at various hydrolysis times. 
All measurements were performed in triplicate for each sample. Results were given as the mean value and standard deviation of $G^{\prime}, G^{\prime \prime}$ and $\delta$ which were calculated from the three determinations.

\section{RESULTS AND DISCUSSIONS}

\subsection{Microsphere synthesis}

Degradable and non degradable microspheres were obtained with good production yield. Indeed, $80 \%$ of the total mass of polymer produced during polymerization occurred as microspheres while only $20 \%$ were discarded as aggregates. Particles were spherical (Figure 2) and their diameter and size distribution were comprised within the range of the size of particles $(315-500 \mu \mathrm{m})$ expected from the sieve used to isolate the microspheres after synthesis (Table I).

Swelling measurements were carried out for non-degradable microspheres with different crosslinking ratios. The swelling of the microspheres greatly depended on the crosslinking agent concentration used during the synthesis (Figure 3). Highly crosslinked microspheres contained less amount of absorbed water while the lower the crosslinking the larger was the swelling. This result agreed with what is generally observed considering bulk hydrogels $[20,21]$.
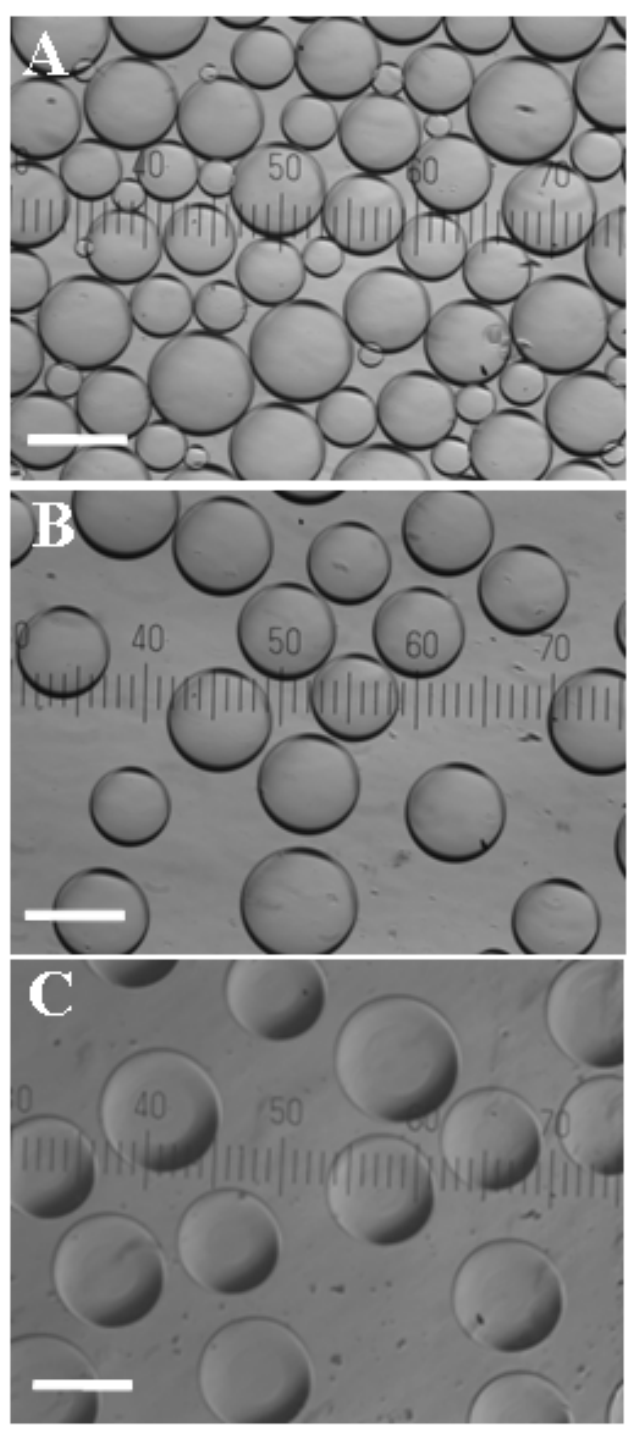

Figure 2: Morphological observations by optical microscopy of microspheres PEGDMA-1\% in different stages of the study. A - Before sieving. B - After sieving 315-500 $\mu \mathrm{m}$, before rheological study. C - After sieving 315-500 $\mu \mathrm{m}$, after rheological study. Scale bar: $400 \mu \mathrm{m}$ 


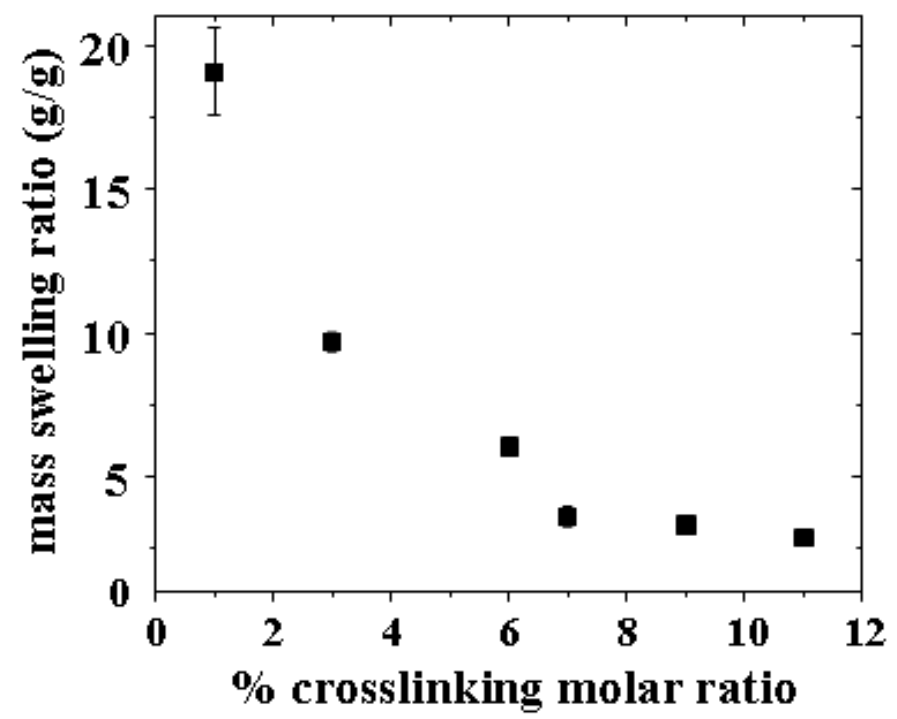

Figure 3: Swelling ratio of microspheres as the function of their crosslinking molar ratio.

\subsection{Development of the rheological method for the characterisation of the microspheres}

The purpose of the work was to develop a rheological method to characterize mechanical properties of hydrogel microspheres while they remained under individual microspheres during the entire process of the measurement. This was in contrast with many works in which the properties of the hydrogel forming microspheres or nanospheres were approached by measuring the rheological properties of a bulk hydrogel of the same composition [22,23]. Preliminary experiments included the determination of the concentration in microspheres of the suspensions used for rheological measurement and determination of the gap between the plates of the measurement cell that needed to be used to provide with relevant and reproducible experimental measurements.

\subsection{Concentration in microspheres in the suspension}

The key parameter for rheological measurements of a suspension is that it must form a homogeneous medium. In physical studies in general and particularly in rheology, the volume fraction is frequently used to characterise the concentration of the dispersed phase in a suspension. It is an important factor which greatly influences the obtained moduli in rheological measurements [24]. This dependence is not only an issue for suspensions but also for bulk hydrogels $[19,25]$. Consequently, it was important to verify that all measurements were performed at the same concentration in microspheres in the suspension throughout the study to be able to obtain relevant comparable results. The volume fraction can be calculated by the ratio of the volumes of all the particles (particles without the liquid entrapped in the void spaces subsisting between microspheres) to the total volume of the suspension (particles plus dispersing liquid). However, volume fraction is sometimes difficult to determine at it requires to know the density of the dispersed particles. This was the case with the hydrogel microspheres investigated in the present study. A related factor can be used in the case which corresponds to the mass fraction. This parameter can be measured from the determination of the weight of the wet microspheres contained in a defined weight of sample. The mass fractions measured for all the suspensions used in this study at their maximal concentration obtained after sedimentation for $30 \mathrm{~min}$ were $65 \pm 3 \%$. As the standard deviation was low between the different samples of microspheres, it was considered that rheological measurements were performed on suspensions at a constant mass fraction of the microspheres. 


\subsection{Choice of the gap between plates of the measurement cell}

Sedimentation occurring during measurement is another parameter influencing results from rheological evaluations performed on microsphere suspensions. To avoid sedimentation phenomenon with the large size hydrogel microspheres dispersed in PBS, we have chosen a gap between plates of the measurement cell in which only one layer of microspheres can fit in (i.e., 300 $\mu \mathrm{m}$ ). The gap was then slightly smaller than the mean size of the microspheres (microspheres sieved between 315 and $500 \mu \mathrm{m}$ ) assuming that the microspheres formed a reproducible homogenous monolayer of material between the plates of the cell measurement. This gap was also chosen to ensure that only the elasticity of the microspheres will be investigated. It was verified that the measurements were conducted in the linear regime. Thus, the microspheres were reversibly deformed as confirmed by the observations under the optical microscope.

\subsection{Rheological characterization of microspheres}

\subsubsection{Shear sweep studies}

First, experimental conditions to perform rheological measurements on microspheres in relevant conditions needed to be identified. It was then verified that the material deposited in the measurement cell underwent reversible deformation during shear solicitations. In general, this condition is fulfilled in the linear regime which corresponds to plateau values of $G^{\prime}$ and $G^{\prime \prime}$ shear moduli as a function of the strain. In a first set of experiments, rheological behaviours of non degradable microspheres were monitored under shear sweep to identify the range corresponding to the linear regime. Figure 4 presents the results of the variation of $G^{\prime}$ and $G^{\prime \prime}$ shear moduli for two of the non degradable microspheres used in this study (Figure 4).


Figure 4 Determination of the range of the linear regime under controlled strain in experiment with non-degradable microspheres with crosslinking molar ratio of $2 \%$ (A) and $6 \%$ (B). $G^{\prime}$ (open symbols) and $G^{\prime \prime}($ closed symbols) moduli were monitored as a function of strain sweeping from 0.001 to $10 \%$. The grey zones correspond to the linear regime and the dotted lines indicate the positions of chosen controlled shear strain value (0.04\%) for following experiments. 
According to the results obtained from these experiments, $G^{\prime}$ and $G^{\prime \prime}$ moduli were stable between values of the strain ranging from 0.02 to $0.3 \%$ indicating the limits of the linear regime for all types of microspheres. The imposed strain, $\gamma$, chosen for exploring the rheological behaviour of the microspheres in the oscillatory shear mode used in the next step of the development of the method was $0.04 \%$. It is noteworthy that this value of controlled strain is very low. It insured non-destructive micro-shear preserving microspheres from being damaged during rheological measurements. This was confirmed by a systematic observation of the microspheres by optical microscopy after rheological measurements. This controlled observation performed on the different microspheres after rheological measurements showed that all types of microspheres remained well spherical and did not exhibited any fracture (All data not shown, See for an example Figure $2 \mathrm{C}$ ).

\subsubsection{Frequency sweep studies}

Controlled strain mode with a constant strain at $0.04 \%$ was applied to study the viscoelastic characteristics of the non degradable microspheres prepared with crosslinking ratios ranging between 1 and $11 \%$. Experiments repeated three times on the same sample of microspheres but by refilling the measurement cells with a new amount of sample always gave values in a low range interval (ranging between 5 to $8 \%$ ). An example of the curves obtained showing the mean value with standard deviation of the storage $\left(G^{\prime}\right)$ and loss $\left(G^{\prime \prime}\right)$ moduli as a function of frequency was plotted in Figure $5 \mathrm{~A}$ for the microspheres PEGDMA-1\%.
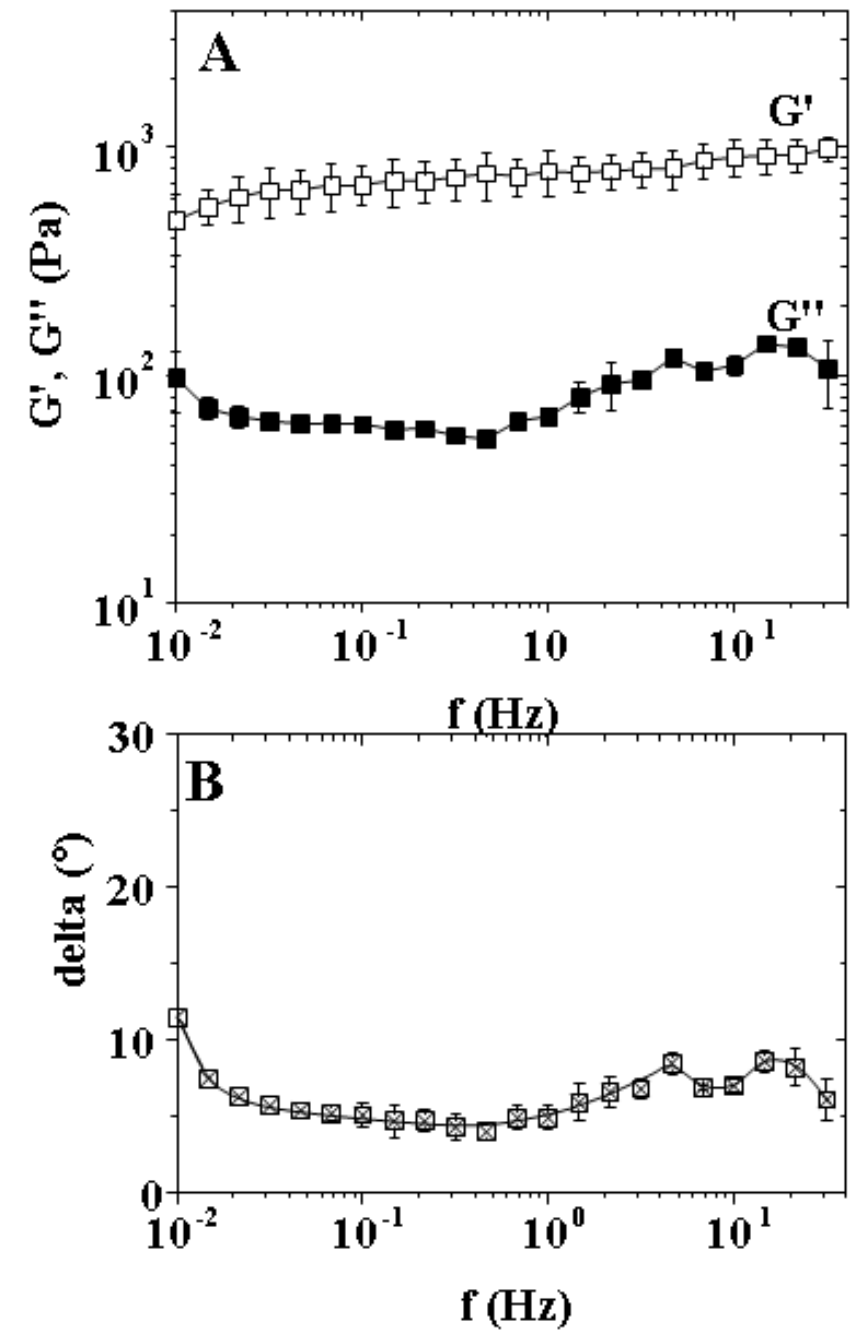

Figure 5 Influence of frequency on the rheological behaviour of the microspheres PEGDMA-1\%. Measurements were performed with the imposed shear strain at $0.04 \%$. A - The storage $\left(G^{\prime}\right)$ and loss $\left(G^{\prime \prime}\right)$ moduli ; $B$ Phase angle $\delta$. 
The two moduli $G^{\prime}$ and $G^{\prime \prime}$ were almost independent on the variation of frequency in the whole range of the frequency sweep. The rheological behaviour showed by the microspheres was typical of that of chemically crosslinked hydrogels. The ratio $G^{\prime \prime} / G^{\prime}$ was roughly in the order of 0.1 and appeared rather independent on the frequency. In agreement with this, the phase angle, $\delta$, was nearly constant over the range of frequencies comprised between 0.05 and $40 \mathrm{~Hz}$ (Figure 5B). In those conditions, the value of the phase angle was around $8^{\circ}$ indicating that the microspheres behaved like a gel material with nearly pure elastic properties.

As shown in Figure 6, the storage modulus, $G^{\prime}$, of the microspheres was almost independent on frequency whatever their crosslinking density was. The value of the storage modulus taken at a frequency of $10 \mathrm{~Hz}$ increased with the degree of crosslinking of the microspheres (Table I, Figure 7).

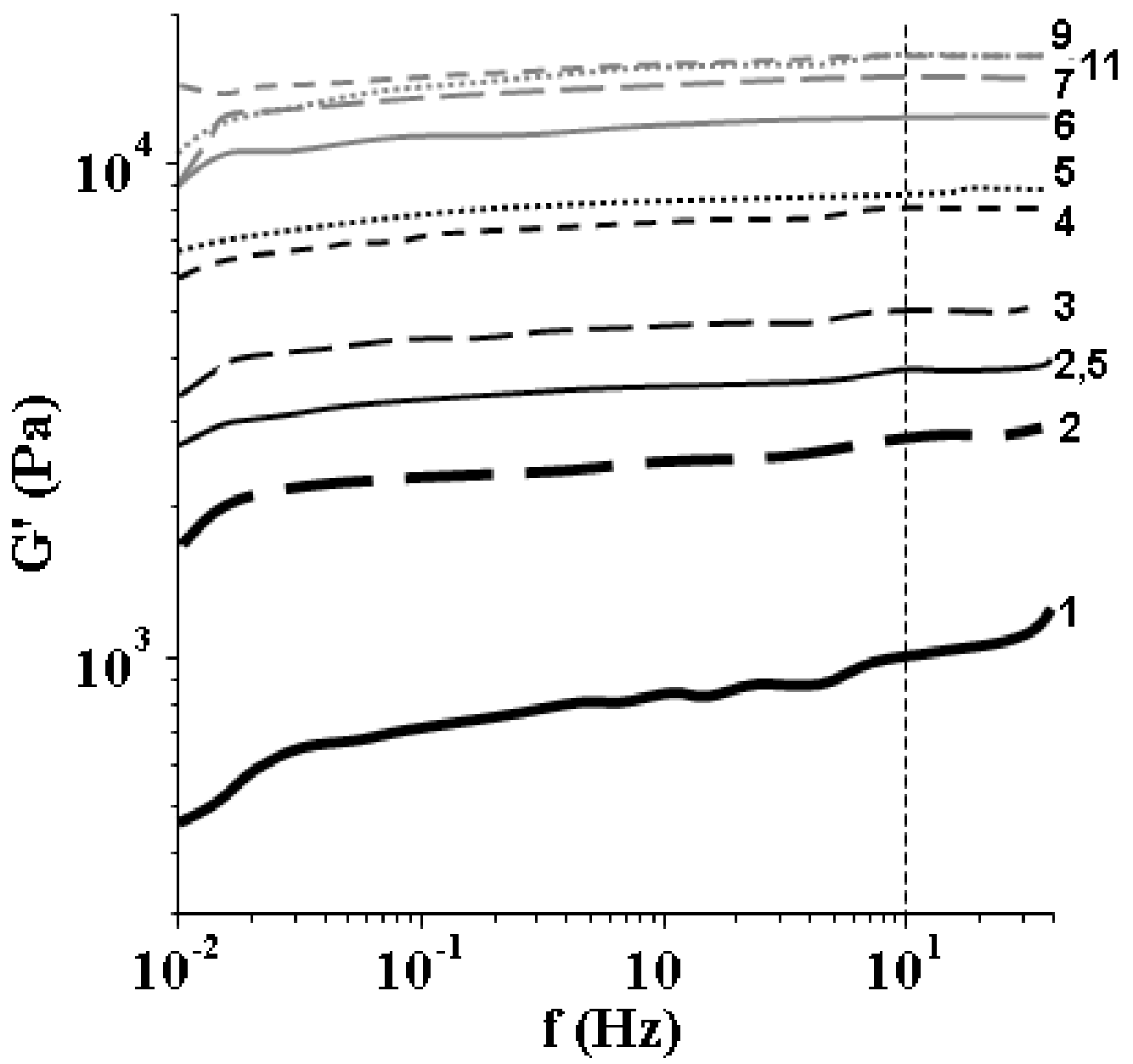

Figure 6 Determination of the storage modulus, $G^{\prime}$, of non-degradable microspheres with different crosslinking molar ratio (thick solid black line $1 \%$, thick long dashed black line $2 \%$, thin solid black line $2.5 \%$, thin long dashed black line 3\%, thin short dashed black line 4\%, dotted black line 5\%, solid grey line $6 \%$, long dashed grey line $7 \%$, short dashed grey line $9 \%$, dotted grey line $11 \%)$ at the oscillatory frequency of $10 \mathrm{~Hz}$ and under an imposed shear strain of $0.04 \%$. 


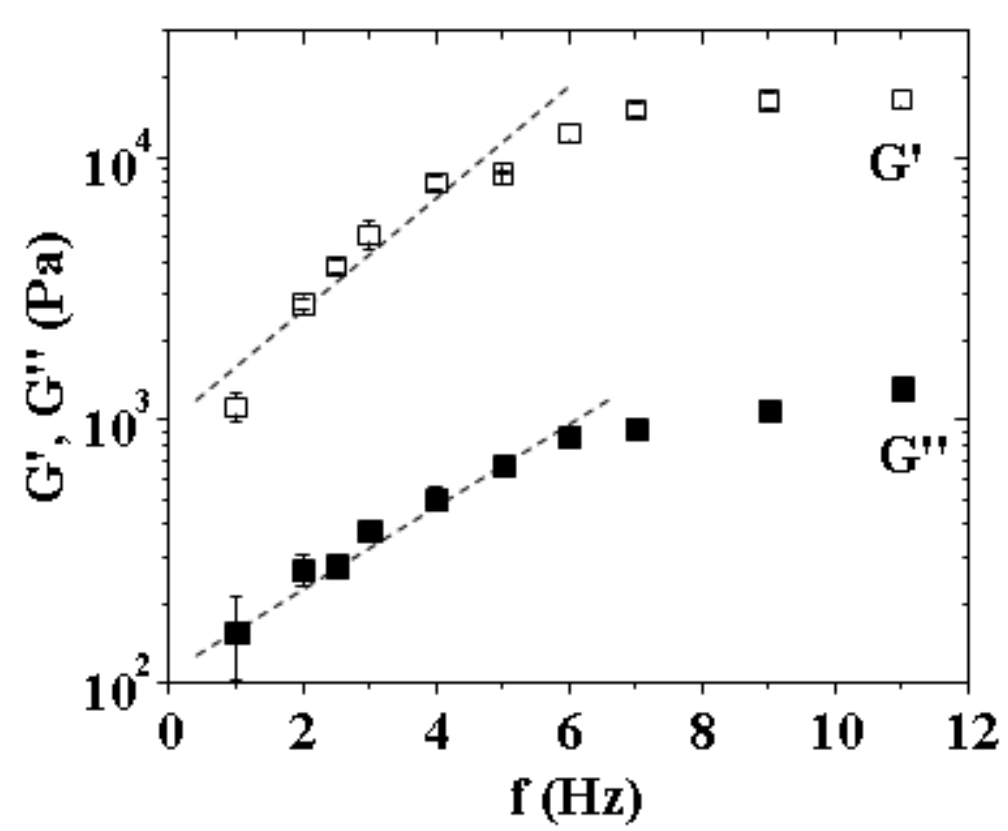

Figure 7 Storage modulus, $G^{\prime}(A)$, and loss modulus, $G^{\prime \prime}(B)$, of hydrogel microspheres with different crosslinking molar ratio. Measurements were performed with an imposed shear strain at $0.04 \%$ and at an oscillation frequency of $10 \mathrm{~Hz}$. The dashed line is only used as a guide to highlight the linear domains of the curve between the storage modulus and the degree of crosslinking of the microspheres.

This was also the case considering the loss modulus, $G^{\prime \prime}$ (Figure 7B). The phase angle deduced from these measurements ranged between 3 to $8^{\circ}$ for the different microspheres (Table I). This indicated that all microspheres showed nearly pure elastic properties.

Both $G^{\prime}$ and $G^{\prime \prime}$ increased linearly with the crosslinking molar ratio of the microspheres up to a crosslinking molar ratio of about $7 \%$. Above this value, $G^{\prime}$ reached a plateau value, while $G^{\prime \prime}$ kept increasing. The results obtained here can be discussed considering the theory of the rubber elasticity proposed by Flory [26] in which the storage modulus, $G^{\prime}$, depends directly on the number of crosslinks per elastically active chain, $v_{e}$, and on a constant characterizing the polymer solvent system, $\alpha$. The relation appearing between these parameters and the storage modulus is given in equation 5.

$G^{\prime}=\alpha v_{e} R T$

where $R$ is the universal gas constant and $T$ the temperature.

The two parameters $\alpha$ and $v_{e}$ are related to the characteristics of a given hydrogel. According to the Flory theory, they represent the swelling capacity of the gel. As a consequence, the above relationship demonstrates the dependence between rheological moduli and swelling ratio of hydrogels. It can be pointed out that the Flory theory applied well with the microspheres considered in the present study. Both the storage modulus, $G^{\prime}$, and the swelling ratio of microspheres with degrees of crosslinking below $7 \%$ varied a lot (Figure 3). In contrast, with highly crosslinked microspheres (crosslinked density above 7\%), very little differences in swelling capacities were observed and the storage modulus reached a plateau value. According to the above results the storage modulus of microspheres varied a lot with crosslinking molar ratio only with microspheres 
crosslinked at a maximum ratio of $7 \%$. Considering these microspheres, an almost linear relation was found between the two parameters (Figure 7A). This suggested that degradation of microspheres through hydrolysis of the crosslink bonds can be monitored by measuring the storage modulus of the microspheres during degradation experiments performed on microspheres with an initial crosslinking molar ratio below $7 \%$.

\subsection{Rheological characterization of the degradation of hydrolysable microspheres}

Degradable microspheres were synthesized using a hydrolysable crosslinker. The degradation of these microspheres was believed to take place by hydrolysis of the crosslinking bonds including lactic and glycolic acid segments occurring with contact of water. Thus, it was expected that the crosslinking density of the microspheres will be reduced during the degradation process which in turn could be monitored by measuring the storage modulus of the microspheres over time. It was also expected that the hydrolysis of the microspheres would be accompanied by the release of carboxylic acids in the incubation medium coming from the hydrolysis of the ester bonds included in the lactic and glycolic acid containing segments of the crosslinker. This effect can be monitored by measuring the $\mathrm{pH}$ of the incubation medium. Thus, an acidification of the incubation medium would signify that the microspheres degraded through the expected hydrolytic mechanism while a decrease of the storage modulus would indicate a loss of crosslinking bonds in the hydrogel structure of the microsphere.

Rheological measurements were performed in conditions established above on microspheres incubated in PBS. In agreement with the work presented in the first part of this paper, microspheres with a low initial crosslinking density (3\%) were selected for this study.

Figure 8 presents the results of the evolution of the $\mathrm{pH}$ of the incubation medium of microspheres HEMA4L4G-3\% monitored during the experiment. It shows a significant decrease in $\mathrm{pH}$ value of the dispersing medium occurring from the start of the experiment up to the $10^{\text {th }}$ day of the incubation time. The decrease of $\mathrm{pH}$ monitored over the first 10 days of the incubation indicated that the microspheres released acidic compounds in the incubation medium. This was in agreement with the hydrolytic mechanism expected from the chemical nature of the hydrogel composing the microspheres. After 10 days, the $\mathrm{pH}$ of the incubation medium remained constant indicating that the degradation of the microspheres was stopped.

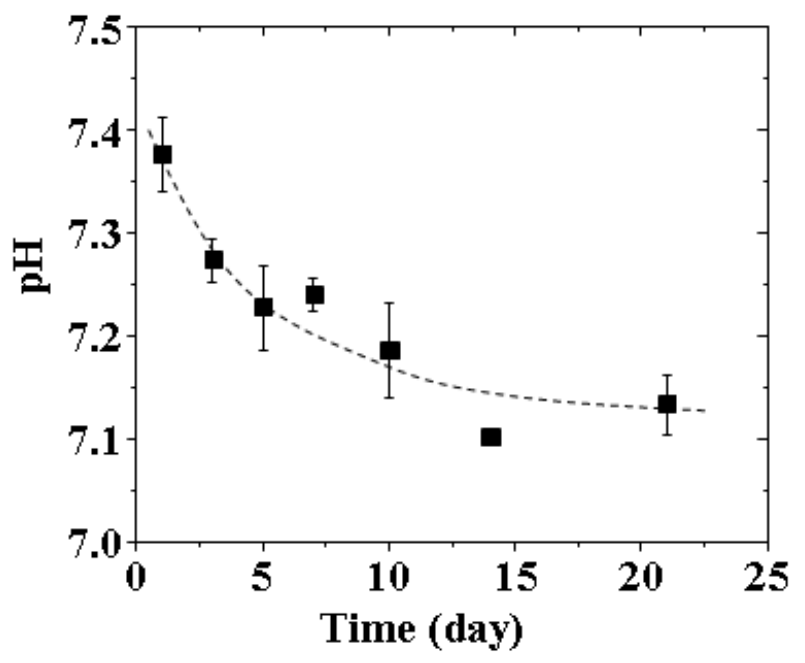

Figure 8 Variation of $p H$ of the incubation medium during incubation of the microspheres in PBS at $37^{\circ} \mathrm{C}$. 
Rheological measurements were performed on degradable HEMA4L4G-3\% microspheres under the experimental conditions defined above: the oscillatory frequency was set to $10 \mathrm{~Hz}$ and the shear strain was set at $0.04 \%$ to remain in the linear regimen. Figure 9 presents the results obtained from the measurement of storage modulus, $G^{\prime}$, of the degradable microspheres HEMA4L4G-3\% incubated in PBS over a period of time of 21 days.
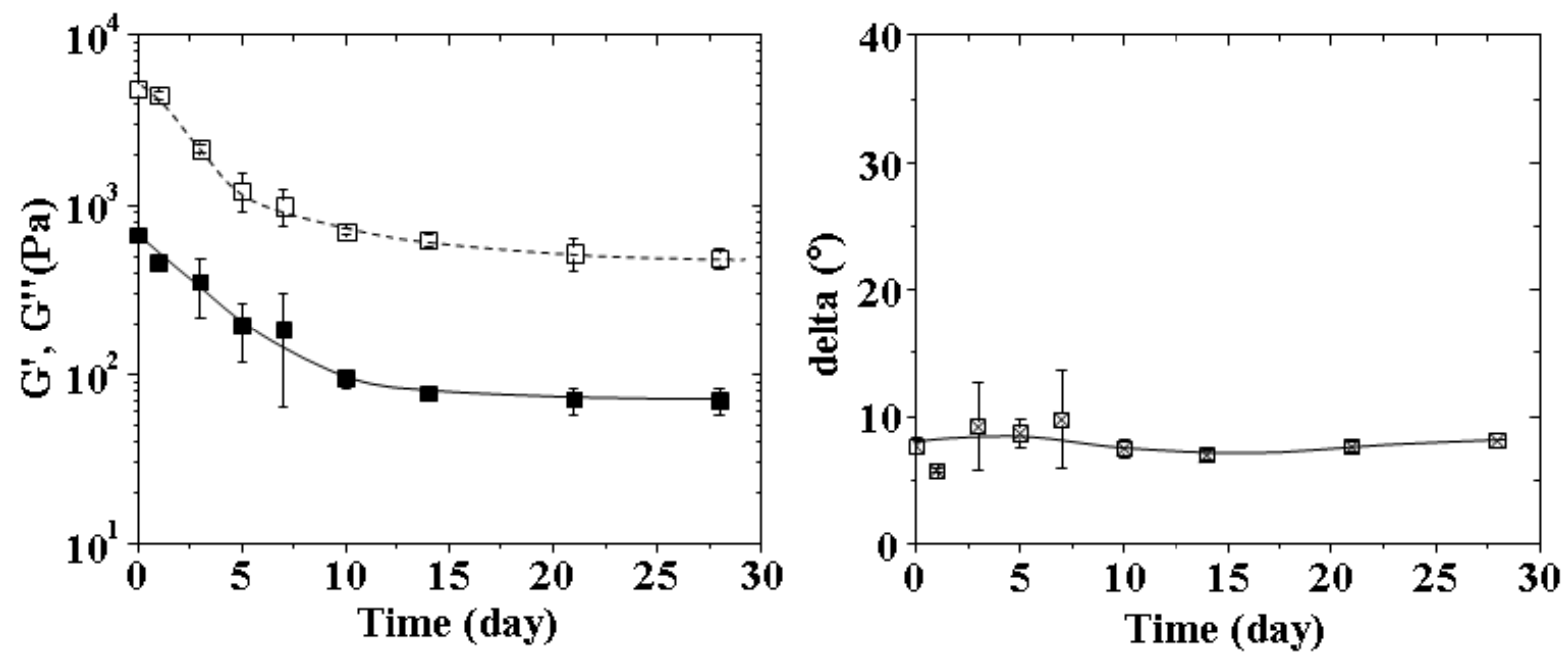

Figure 9 Evolution of rheological properties of microspheres HEMA4L4G-3\% during incubation in PBS at $37^{\circ} \mathrm{C}$. A - Storage $\left(G^{\prime}\right.$, open symbols) and loss $\left(G^{\prime \prime}\right.$, closed symbols) moduli. $B$ - Phase angle $\delta$. Experimental conditions for the measurement were set with an oscillatory frequency at $10 \mathrm{~Hz}$ and a shear strain at $0.04 \%$. The lines were drawn to serve as guides for the eyes.

A clear decrease of $G^{\prime}$ was monitored between the start of the experiment (day 0 ) and day 10 where $G^{\prime}$ reached a plateau value. The loss modulus, $G^{\prime \prime}$, followed exactly the same tendency with $\mathrm{pH}$ over the same period of time. The value of the phase angle, $\delta$, remained constant and close to $8^{\circ}$ during the whole period of time of the experiments indicating that the hydrogel remained as an almost purely elastic material.

The fact that the storage modulus of the microspheres was decreased with time during the incubation of the microspheres in PBS was in favour with a decrease of the crosslinking density of the hydrogel forming the microspheres. The time scale in which the modification of the rheological properties of the microspheres occurred correlated well with the time scale during which the $\mathrm{pH}$ of the incubation medium dropped down. This last result suggested that the modification of the rheological properties monitored during the incubation of the microspheres in PBS resulted from the degradation of the hydrogel through the hydrolysis of the crosslinking bonds.

\section{CONCLUSION}

Conditions to measure rheological properties of microspheres made of a chemically crosslinked hydrogel and remaining as individual microspheres were established. It was determined that relevant and reproducible measurements could be obtained with a rheometer equipped with a parallel plate geometry in which a homogenous and continuous monolayer of microspheres is inserted in the gap between the plates. The storage moduli of the microspheres followed the theory of rubber elasticity developed by Flory. It was affected by the degree of crosslinking of the hydrogel forming the microspheres in the same crosslinking density range than that affected the swelling ratio of the 
microspheres. Results from this work also showed that degradation of hydrolysable crosslinked microspheres can be monitored by measuring their storage modulus over time by applying the rheological method of characterization of microspheres developed in this work. Further experiments will be conducted on hydrolysable microspheres to investigate the influence of the crosslinking molar ratio and of the nature of the degradable crosslinking bond on both the rheological properties of the microspheres and their degradation rate.

\section{ACKNOWLEDGEMENTS}

The authors would like to thank CNRS and University of Paris Sud. The authors would also like to thank G. MEKHLOUFI for her help in performing and analysing the size of the microspheres.

\section{REFERENCES}

1. Ratner, B. D.; Hoffman, A.S. (1976). "Synthetic Hydrogels for Biomedical Applications. Hydrogels for Medical and Related Applications." Andrades, J.D.; Ed.; American Chemical Society, Washington DC, 1976, Chapter 1, pp 1-36.

2. Peppas, N. A.; Bures, P.; Leobandung, W.; Ichikawa, H. "Hydrogels in pharmaceutical formulations." Eur. J. Pharm. Biopharm. 2000, 50, 27-46.

3. Hoffman, A. S. "Hydrogels for biomedical applications." Adv. Drug Deliv. Rev. 2002, 54, 3-12.

4. Kopecek, J. "Polymer chemistry: swell gels." Nature 2002, 417, 388-389, 391.

5. Caldorera-Moore, M.; Peppas N.A. "Micro- and nanotechnologies for intelligent and responsive biomaterial-based medical systems." Adv. Drug Deliv. Rev. 2009, 61, 1391-1401.

6. Kopecek, J. "Hydrogels: From soft contact lenses and implants to self-assembled nanomaterials." J. Applied Polym. Sci. Part A; Polym. Chem. 2009, 47, 5929-5946.

7. Elbert, D. L. Liquid-liquid two-phase systems for the production of porous hydrogels and hydrogel microspheres for biomedical applications: A tutorial review. Acta Biomater. 2011, 7,31-56.

8. Tajima, S.; Tabata, Y. Preparation and functional evaluation of cell aggregates incorporating gelatin microspheres with different degradabilities. J. Tissue Eng. Regen. Med. 2012 doi: 10.1002/term.1469.

9. Garg, T.; Singh, O.; Arora, S.; Murthy, R. Scaffold: a novel carrier for cell and drug delivery. Crit Rev Ther Drug Carrier Syst. 2012, 29,1-63.

10.Vermonden, T.; Besseling, N. A. M.; van Steenbergen, M. J.; Hennick, W. E. "Rheological Studies of Thermosensitive Triblock Copolymer Hydrogels." Langmuir 2006, 22, 10180-10184.

11.Moura, M. J., Figueiredo, M. M.; Gil, M. H. "Rheological Study of Genipin Crosslinked Chitosan Hydrogels." Biomacromolecules 2007, 8, 3823-3829.

12.Neamtu, I.; Nita, L. E.; Bercea, M.; Chiriac, A. P. "The rheological behaviour of chemically crosslinked hydrogels based on poly(acrylamide)." Polymer 2009, 54, 795-800.

13.Payet, L.; Ponton, A.; Grossiord, J.-L.; Agnely, F. "Structural and rheological properties of chitosan semi-interpenetrated networks" Eur. Phys. J. 2010, E 32, 109-118.

14.Khalid, M.N.; Ho, L.; Agnely, F.; Grossiord, J.L.; Couarraze G. "Swelling properties and mechanical characterization of a semi-interpenetrating chitosan/polyethylene oxide network. Comparison with a chitosan reference gel" S.T.P. Pharma. Sci. 1999, 9, 359-364.

15.Gever, L. M.; Lyons, J. G.; Higginbotham, C. L. "Photopolymerisation and characterisation of negative temperature sensitive hydrogels based on N,N-diethylacrylamide." J. Mater. Sci. 2001, 46, 509-517.

16.Zustiak, S. P.; Leach, J. B. "Hydrolytically Degradable Poly(Ethylene Glycol) Hydrogel Scaffolds with Tunable Degradation and Mechanical Properties." Biomacromolecules 2010, 11, 1348-1357. 
17.Potta, T.; Chun, C.J.; Song, S-C. "Chemically crosslinkable thermosensitive polyphosphazene gels as injectable materials for biomedical applications." Biomaterials 2009, 30, 6178-6192.

18.Van Tomme, S. R.; van Nostrum, C. F.; de Smedt, S. C.; Hennick, W. E. "Degradation behaviour of dextran hydrogels composed of positively and negatively charged microspheres." Biomaterials 2006, 27, 4141-4148.

19. Raquois, C.; Tassin, J. F.; Rezaiguia, S.; Gindre, A. V. "Microgels in coating technology: structure and rheological properties." Prog. Organic Coating 1995, 26, 239-250.

20.Ibrahim, S.; Kang, Q. K.; Ramamurthi A. "The impact of hyaluronic acid oligomer content on physical, mechanical, and biologic properties of divinyl sulfone-crosslinked hyaluronic acid hydrogels." J. of Biomed. Mater. Res. Part A, 2010, 94A, 355-370.

21.Wang, K.; Xu, X.; Liu, T. T.; Fu, S. Z.;Guo, G.; Gu, Y. C.; Luo, F.; Zhao, X. Wei, Y.Q.; Qian, Z. Y. "Synthesis and characterization of biodegradable $\mathrm{pH}$-sensitive hydrogel based on poly(ecaprolactone), methacrylic acid, and Pluronic (L35)" Carbohydrate Polymers 2010, 79, 755-761.

22.Hu, X.; Zhou, J.; Zhang, N.; Tan, H.; Gao, C. "Preparation and properties of an injectable scaffold of poly(lactic-co-glycolic acid) microparticles/chitosan hydrogel." J Mech. Behav. Biomed. Mater. 2008, 1, 352-359.

23.Kumachev, A.; Greener, J.; Tumarkin, E.; Eiser, E.; Zandstra, P. W.; Kumacheva, E. "Highthroughput generation of hydrogel microbeads with varying elasticity for cell encapsulation." Biomaterials 2011, 32, 1477-1483.

24.Taylor, K. W.; Bagley, E. B. "Rheology of Dispersions of Swollen Gel Particles." J. Polym. Sci. Polym. Phys. Ed. 1975, 13, 1133-1144

25.Baker, B.; Murff, R.; Milam, V. T. "Effects of volume fraction and composition on the rheological properties of polyacrylic acid/polyacrylamide hydrogels." Mater. Res. Soc. Symp. Proc. 2007, 975, \#0975-DD06-06.

26. Flory; P. J. "Principle of polymer chemistry", Cornell University Press, Ithaca, New York, 1953. 